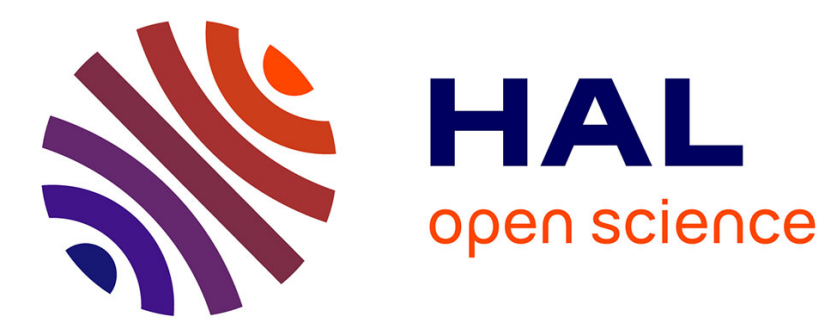

\title{
Filter-less submicron hydrodynamic size sorting
}

Marc Fouet, Maud-Alix Mader, Sabri Iraïn, Zineb Yanha, Antoine Naillon, Sébastien Cargou, Anne Marie Gué, Pierre Joseph

\section{To cite this version:}

Marc Fouet, Maud-Alix Mader, Sabri Iraïn, Zineb Yanha, Antoine Naillon, et al.. Filter-less submicron hydrodynamic size sorting. Lab on a Chip, 2016, 16 (4), pp.720-733. hal-01222327v3

\section{HAL Id: hal-01222327}

https://hal.science/hal-01222327v3

Submitted on 25 Jan 2016

HAL is a multi-disciplinary open access archive for the deposit and dissemination of scientific research documents, whether they are published or not. The documents may come from teaching and research institutions in France or abroad, or from public or private research centers.
L'archive ouverte pluridisciplinaire HAL, est destinée au dépôt et à la diffusion de documents scientifiques de niveau recherche, publiés ou non, émanant des établissements d'enseignement et de recherche français ou étrangers, des laboratoires publics ou privés. 


\title{
Filter-less submicron hydrodynamic size sorting
}

\author{
M. Fouet ${ }^{\ddagger},{ }^{a, b}$ M.-A. Mader ${ }^{\ddagger},{ }^{a, b, c}$ S. Iraïn, ${ }^{a, b}$ Z. Yanha, ${ }^{a, b}$ A. Naillon, ${ }^{a, b, d, e}$ S Cargou, ${ }^{a, b}$ A.-M. Gué, ${ }^{a, b}$ and \\ P. Joseph, ${ }^{a, b^{*}}$ \\ ${ }^{a}$ CNRS, LAAS, 7 avenue du colonel Roche, F-31400 Toulouse, France. \\ ${ }^{b}$ Univ de Toulouse, UPS, LAAS, F-31400 Toulouse, France. \\ ${ }^{c}$ RTRA-STAE, 23 avenue Edouard Belin, F-31400 Toulouse, France. \\ ${ }^{d}$ INPT, UPS, IMFT, Université de Toulouse, Allée Camille Soula, F-31400 Toulouse, France \\ ${ }^{e}$ CNRS, IMFT, F-31400 Toulouse, France \\ These two authors made equal contributions. \\ ${ }^{*}$ Corresponding author.
}

\begin{abstract}
We propose a simple microfluidic device able to separate submicron particles (critical size $\sim 0.1 \mu \mathrm{m}$ ) from a complex sample with no filter (minimum channel dimension being $5 \mu \mathrm{m}$ ), by hydrodynamic filtration. A model taking into account the actual velocity profile and hydrodynamic resistances enables predicting the chip sorting properties for any geometry. Two design families are studied to obtain (i) small sizes within minutes (low-aspect ratio, two-level chip) and (ii) micron-sized sorting with $\mu \mathrm{L}$ flowrate (3D architecture based on lamination). We obtain quantitative agreement of sorting performances both with experiments and with numerical solving, and determine the limits of the approach. We therefore demonstrate a passive, filter-less sub-micron size sorting with simple, robust, easy to fabricate design.
\end{abstract}

\section{A. Introduction}

Microfluidic systems constitute great tools for analytical chemistry, in particular when dealing with preparation of samples. In this area, a lot has been done for cell biology (cell sorting ${ }^{1-3}$, blood analysis ${ }^{4-7}$ ) or molecular biology (micro/nanochannels interface for preconcentration of proteins for instance $^{8,9}$ ).

Let us take two examples for which progress in sample preparation are still strongly awaited for. First, in the context of environmental science, the demand for systems suited for the handling of real, complex samples, such as rivers waters for the detection of pollutants, is growing rapidly. For instance, mercury is a highly toxic element, and its monitoring in rivers is thus necessary ${ }^{10}$. However, a single value of the total amount of mercury is not sufficient, because (i) mercury toxicity strongly depends on its speciation (chemical form), and (ii) transport of the pollution is strongly affected by the size of the carrying sediment. Therefore, correlating size and concentration gives information on the pollution extent that is much more relevant than a simple titration. As second example, microparticles in the blood (50-1000 nm vesicles originating from cell membranes) have recently been identified as particularly relevant biomarkers for cancers and many diseases (autoimmune, inflammatory, infectious, cardiovascular $)^{11}$; extracting those small objects from a blood sample is thus of strong interest for diagnostics.

A particularly important function of sample preparation is thus fractionation, defined as the sorting by size independently of other physical and chemical parameters.

Numerous innovative approaches have been recently developed to sort particles in microfluidic devices ${ }^{12,13}$. Sorting methods are usually classified in two groups: active and passive ones. Active approaches require external force, originating for example from acoustic fields (acoustophoresis ${ }^{14}$ ), electric fields (dielectrophoresis ${ }^{15,16}$ or AC electrophoresis), magnetic fields ${ }^{17}$ or optical fields ${ }^{18}$, and 
are consequently sensitive not only to particle size, but also to other property such as charge, polarizability or density. Passive methods involve only internal forces and channel design and are thus better suited for purely size sorting. In addition, passive methods are more likely not to interfere with downstream detection principle (an electric field could modify for example an electrochemical equilibrium).

However, standard passive separation principles are not fully suited for in situ devices. Among them, centrifugation is extensively used but remains very difficult to integrate on a device (with the notable exception of lab-on-disc ${ }^{19-21}$ ), and almost impossible to operate continuously. Let us also note that separation criterion is not strictly based only on size, since particle density is also involved. The approach based on inertial forces ${ }^{4}$, developed by Di Carlo and colleagues is very appealing because it does not necessitate a strong confinement. It is however not suited for sub-micrometric particles because moderate particles Reynolds number is required for the separation mechanism to be efficient. The $\mathrm{H}$ sorter as earlier proposed by P. Yager ${ }^{22,23}$ relies on diffusion mechanisms at the interface of laminar flows and is therefore much more adapted to molecular species separation than to particle sorting.

The most intuitive fractionation principle relies on filtration by a membrane, which can be quite efficient and selective. Many efforts have been made recently for integrating membranes inside microfluidic devices ${ }^{24-26}$. However, this approach is inherently subject to pore obstruction, leading to poisoning, and should be avoided for applications targeting in situ applications. The "deterministic lateral displacement" is a microfluidic technique using physical obstacles ${ }^{27}$ that has gained much attention in the last years ${ }^{28}$. However, obstacles should be discarded for in situ analysis because they are prone to clogging, similarly to the use of a membrane. Indeed the gap between two obstacles is usually close to the size of the analyte particle (minimum sorting diameter of order one fifth of the $\left.\operatorname{gap}^{27,28}\right)$.

A simple, passive sorting method has been proposed and demonstrated a few years ago by Seki and Yamada $^{29,30}$, that they baptized hydrodynamic filtration. Its principle, as explicated in Figure 1, is based on the exclusion of large objects from the streamlines located close to a wall: after a bifurcation between a main channel and a small lateral one, the lateral output channel contains only small particles. The width $r_{c}$ of the portion of fluid flowing through the side channel defines the critical sorting size of the branching: particles outside this zone are excluded from the lateral channel. A particle flowing next to the sidewall cannot enter the side channel if its radius is bigger than $r_{c}$ (corresponding to particle size $2 r_{c}$ ). For given outlets pressures (in general both set equal to the atmospheric pressure), the distribution of flow rates at a branch point, which determines the size limit of the filtered particles, is controlled by the ratio of the outlets hydraulic resistances, so that designing the micro channel geometry enables reaching the desired sorting size.

a)

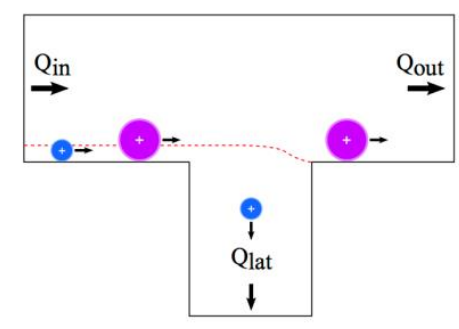

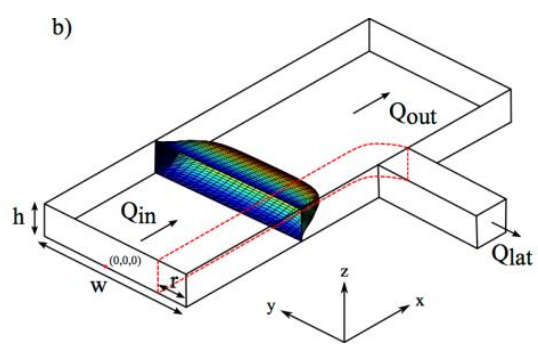

c)

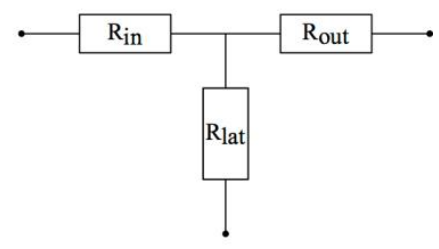

Figure 1- Hydrodynamic filtration. (a) Principle (top view); (b) 3-dimensional scheme of the separation: velocity profile in the inlet channel, notations for the axis and flowrates. The red dashed line is the separation curve; (c) Equivalent electrical circuit.

The hydrodynamic filtration method answers an essential constraint related to robustness (critical, for example, for in situ sample preparation): it is less prone to clogging than filter-based geometries, since the minimal dimension of channels can be more than one order of magnitude superior to the critical sorting size.

Nevertheless, such microfluidic integrated fractionation has only been demonstrated for quite big particles (diameter superior to about one micron). ). As a matter of fact current planar structures 
feature numerous limitations or drawbacks (mainly large footprint, high residence time), and the current modelling relies on a parabolic approximation of the velocity profile which is not valid in many real configurations of channels (varying aspect ratio).

Revisiting this approach, we propose two original configurations (two-level design and 3D networks of channels) in order to sort sub-micronic particles. In this article, we introduce an exact model based on the real shape of the velocity profile for a rectangular shaped channel. Then, we introduce a new two-level design. It allows drastically reducing the total channel length needed for sub-micron sorting which facilitates the fabrication of the device, reduces its footprint, and makes possible its application for reasonable pressures (a fraction of Bar) and residence times (a few minutes). We experimentally validate the extension to the sub-micron regime (exclusion of $\sim 200 \mathrm{~nm}$ diameter particles). We also experimentally demonstrate the efficiency of an original 3D configuration, suitable to extract a larger fraction of fluid with similar sorting size. We obtain a quantitative agreement with our model, both in 2D and 3D configurations, demonstrating its accuracy for rectangular channels of different aspect ratios. Finally, we assess the limits of the analytical model by (i) determining numerically the actual shape of the exclusion zone boundary, and (ii) quantifying the effects of Brownian motion on the exclusion process.

\section{B. Extended model of hydrodynamic filtration}

In their pioneering work demonstrating the original concept of hydrodynamic filtration ${ }^{29}$, Yamada and Seki use microchannels with square cross section (aspect ratio, defined as height/width, equal to unity), parabolic approximation for the velocity profile, and critical separation size of order one micron.

In order to adapt the devices to the separation of much smaller particles, while keeping the smallest dimension in the $10 \mu \mathrm{m}$ range (which enables to avoid filter or membrane, limitative for many applications, as already mentioned, and prevents from reducing too much the flowrate), it is desirable to extend the analysis to more generic geometries.

Thus, we need to generalize demonstration of the critical separation size. To do so, we introduce the main ingredients of hydrodynamic filtration.

First, when a solution is injected inside a microchannel, with typical values of flow rate and pressure drop, Reynolds number is of order unity or lower, and flows are laminar. The Navier-Stokes equation reduces to the Stokes equation, which is linear. For a pressure-driven flow, and a non-compressible Newtonian fluid, the flow profile is often considered to be parabolic (hypothesis used in ${ }^{29,30}$ ), but this approximation is only valid along the smallest dimension. For a rectangular channel of arbitrary aspect ratio $h / w$ (where $w$ and $h$ are the channel width and height respectively, see Figure 1(b)), the velocity profile can be solved exactly and expressed as a Fourier series (for a complete demonstration, see the textbook from Bruus $\left.{ }^{31}\right)$, with the notations of Figure 1(b):

$$
v(y, z)=\frac{4 h^{2} \Delta P}{\pi^{3} \eta L} \sum_{n, o d d}^{\infty} \frac{1}{n^{3}}\left[1-\frac{\cosh \left(n \pi \frac{y}{h}\right)}{\cosh \left(n \pi \frac{w}{2 h}\right)}\right] \sin \left(n \pi \frac{z}{h}\right),
$$

where $\Delta \mathrm{P}$ is the pressure difference applied on the length $\mathrm{L}$ of the channel, and $\eta$ is the dynamic viscosity of the liquid.

Integrating the velocity over channel cross section gives the total input flow rate $\mathrm{Q}_{\text {in }}$ :

$$
Q_{\text {in }}=\frac{h^{3} w \Delta P}{12 \eta L}\left[1-\sum_{n, o d d}^{\infty} \frac{1}{n^{5}} \frac{192}{\pi^{5}} \frac{h}{w} \tanh \left(n \pi \frac{w}{2 h}\right)\right] .
$$

Since there is a linear relationship between pressure drop $\Delta \mathrm{P}$ and flow rate $\mathrm{Q}$, this equation enables introducing the hydraulic resistance $\mathrm{R}_{\mathrm{h}}=\Delta \mathrm{P} / \mathrm{Q}$, which only depends on geometry. For a rectangular channel, it thus reads:

$$
R_{h}=\frac{12 \eta L}{w h^{3}} /\left[1-\sum_{n, o d d}^{\infty} \frac{1}{n^{5}} \frac{192}{\pi^{5}} \frac{h}{w} \tanh \left(n \pi \frac{w}{2 h}\right)\right] .
$$


Let us consider the situation of a simple branching, with the geometry and notations of Figure 1(c), where the objective is to extract only small particles in the lateral outlet (denoted 'lat'), whereas all particles can flow through the other exit (denoted 'out'). For incompressible fluids, the input volumetric flow rate is the sum of the exiting flow rates: mass conservation reads $Q_{\text {in }}=Q_{\text {lat }}+Q_{\text {out }}$.

$Q^{*}=Q_{\text {lat }} / Q_{\text {in }}$, the ratio of the lateral $\left(Q_{\text {lat }}\right)$ to the inlet $\left(Q_{\text {in }}\right)$ flowrates, depends both on the channels resistances and applied pressures. Using Ohm's law and injecting Millman's theorem (applied to the pressure at the intersection) into mass conservation, we can express $Q^{*}$ :

$$
Q^{*}=\frac{R_{\text {out }}+R_{\text {in }}\left(1-\Delta P^{*}\right)}{R_{\text {out }}+R_{\text {lat }} \Delta P^{*}}, \text { with } \Delta P^{*}=\frac{P_{\text {in }}-P_{\text {out }}}{P_{\text {in }}-P_{\text {lat }}} \text {. }
$$

In most situations, the two exit channels are submitted to the same pressure drop (for instance if they are both connected to reservoirs at atmospheric pressure, $\Delta P^{*}=1$ ), so that $\mathrm{Q}_{\text {lat }} \cdot \mathrm{R}_{\text {lat }}=\mathrm{Q}_{\text {out }} \cdot \mathrm{R}_{\text {out }}$, and we can express the flow rate ratio at the intersection with the simplified expression:

$$
Q^{*}=\frac{Q_{\text {lat }}}{Q_{\text {in }}}=\frac{1}{1+\frac{R_{\text {lat }}}{R_{\text {out }}}}=\frac{R_{\text {out }}}{R_{\text {out }}+R_{\text {lat }}} .
$$

The ratio $Q^{*}$ of the flow rate into the lateral channel $\left(Q_{\text {lat }}\right)$ to the total flow rate introduced into the branch point $\left(Q_{i n}\right)$ is thus fully determined by the exit resistances $R_{\text {lat }}$ and $R_{\text {out. For rectangular }}$ channels, it can be computed exactly according to Equation 3.

The sorting radius $r_{c}$ is given by the position of the critical streamline that separates the flow at the bifurcating channel (see Figure 1(a)). Indeed, $r_{c}$ is equal to the distance of this line to the wall. This position is related to two elements:

(i) The fraction $Q^{*}$ of fluid pumped inside the lateral channel, fixed by the ratio of output/lateral resistances, as demonstrated above (see Equation 5);

(ii) The shape of the velocity profile in the upstream channel (Equation 1) since it determines the relation between the partial flow rate $Q_{\text {lat }}$ and the width $r_{c}$ of the fluid vein exiting in the lateral channel.

Consequently, in order to relate the position of the critical streamline in the input channel to $Q^{*}$, we also need to compute the partial flow rate between the wall and a given distance from the wall. We have done it analytically by integrating Equation 1 over a fraction of the channel: on its total height for the $\mathrm{z}$ direction, and up to a distance $\mathrm{r}$ from the wall, see Figure 1(b). The partial flow rate is:

$$
Q(r)=\int_{z=0}^{h} \int_{y=-\frac{w}{2}}^{-\frac{w}{2}+r} v(y, z) d y d z,
$$

which after taking into account Equation 1 reads:

$$
Q(r)=\frac{h^{3} w \Delta P}{12 \mu L} \frac{r}{w}\left[1-\sum_{n, o d d}^{\infty} \frac{1}{n^{5}} \frac{96}{\pi^{5}} \frac{h}{r}\left[\tanh \left(\frac{n \pi w}{2 h}\right)\left(1-\cosh \left(\frac{n \pi r}{h}\right)\right)+\sinh \left(\frac{n \pi w}{2 h}\right)\right]\right] .
$$

The fraction of fluid flowing in a layer comprised within a distance $r$ from the lateral wall can thus be expressed, by combining Equations 2 and 7, as:

$$
Q^{*}=\frac{r}{w} \frac{1-\sum_{n, o d d}^{\infty} \frac{196 h}{n^{5} \pi^{5}}\left[\tanh \left(\frac{n \pi w}{2 h}\right)\left(1-\cosh \left(\frac{n \pi r}{h}\right)\right)+\sinh \left(\frac{n \pi w}{2 h}\right)\right]}{\left[1-\sum_{n, o d d}^{\infty} \frac{1192 h}{n^{5} \pi^{5} w} \tanh \left(\frac{n \pi w}{2 h}\right)\right]} .
$$

Interestingly, Equation 8 shows that the normalized partial flow rate $\mathrm{Q}^{*}$, which is a function of the three lengths $\mathrm{h}, \mathrm{w}$ and $\mathrm{r}$, can in fact be expressed as two ratios of those variables. The variation of $\mathrm{Q}^{*}$ versus the position of the separating streamline adimensionalized by channel width (reduced variable $\mathrm{r} / \mathrm{w}$ ) is plotted in Figure 2(a) for different values of the aspect ratio of the input channel $\mathrm{h} / \mathrm{w}$. 

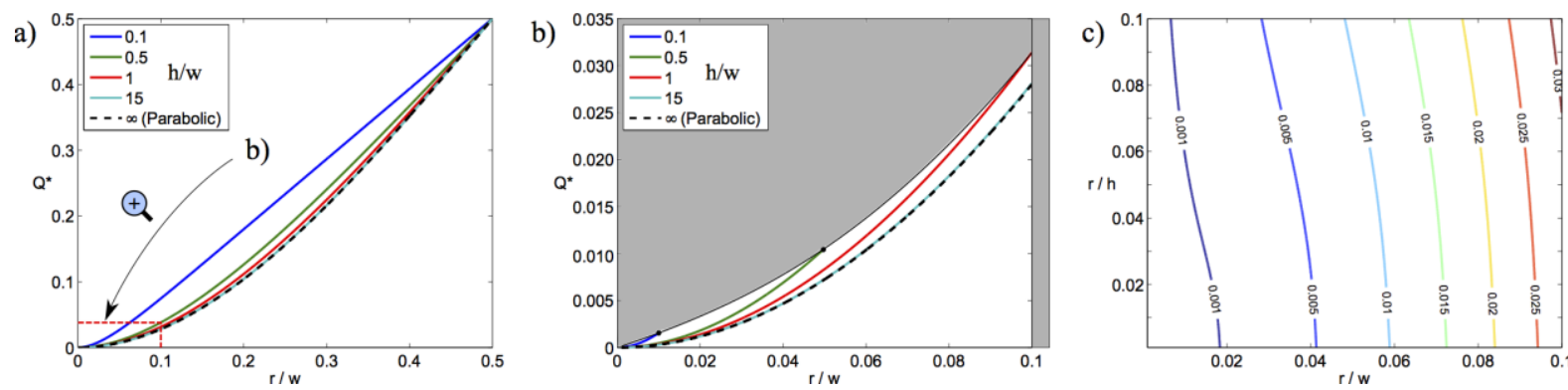

Figure 2- Influence of the geometry on the normalized partial flow rate $\mathrm{Q}^{*}$ (ratio lateral/input flow rate). (a) $\mathrm{Q}^{*}$ plotted as function of the position of the separating stream line adimensionalized by channel width $\mathrm{r} / \mathrm{w}$ and parameterized by the aspect ratio $\mathrm{h} / \mathrm{w}$. Dotted black line corresponds to the limit of very high aspect ratio $\mathrm{h} / \mathrm{w} \rightarrow \infty$, for which velocity profile is parabolic along the y-axis and $\boldsymbol{Q}_{\text {para }}^{*}=(\boldsymbol{r} / \boldsymbol{w})^{2}[\mathbf{3}-\mathbf{2 r} / \boldsymbol{w}]$. (b) Zoom of the same graph for values of $\mathrm{r} / \mathrm{w}$ inferior to 0.1 . The filter-less criterion is materialized by the gray zones (see text for details). (c) Contour plot of $\mathrm{Q}^{*}$, with the reduced variables $\mathrm{r} / \mathrm{w}$ and $\mathrm{r} / \mathrm{h}$.

We can now use the two expressions of the partial flow rate (Equations 5 and 8 ) as a tool to define microchannels geometry for any required value of the critical radius according to the following steps.

For a desired sorting size $2 r_{c}$, we can deduce the flow rate ratio from Equation 8 (equivalently read it on Figure 2), at a chosen aspect ratio $\mathrm{h} / \mathrm{w}$ of the input channel. This value of $\mathrm{Q}^{*}$ defines the needed ratio of hydrodynamic resistances for the lateral and output channels, according to Equation 5. A suitable design of the output and lateral branches can then be chosen, by using for example Equation 3 for rectangular channels.

Before using this model to study the role of channels geometry in next section, let us note that it is strictly valid only for particles at sufficiently low volume fraction (so that there is no hydrodynamic interaction between them, and fluid viscosity is not affected too much by the presence of particles). It is also in principle limited to the cases where the hydrodynamic resistance is useable: stationary flows, and channels with length much longer than their width and height. Those conditions are not very restrictive since they are met in most microfluidic applications.

\section{Design considerations}

We now discuss how the design is related to the sorting capability of the device.

(i) High aspect ratio limit. For high aspect ratio $(h / w \gg 1)$, the velocity profile is parabolic along the y-axis, it reads:

$$
v_{\text {para }}(y)=\frac{\Delta P}{3 \eta L} w^{2}\left(1-(2 y / w)^{2}\right) .
$$

The normalized partial flow rate in this case can be written as:

$$
Q_{\text {para }}^{*}=(r / w)^{2}[3-2 r / w] .
$$

This expression is plotted in Figure 2(a-b) (dotted line). As it could be expected, the exact solution (Equation 8) converges towards this limit case (parabolic, $\mathrm{h} / \mathrm{w} \rightarrow \infty$ ), for large values of $\mathrm{h} / \mathrm{w}$. It is noteworthy that even for moderate values of $h / w$ (for example $h / w=1$, corresponding to a square channel), the parabolic approximation is already a quite good estimate as regards the partial flow rate $Q^{*}$.

(ii) Filter-less criterion and maximization of $Q^{*}$. The graph in Figure 2(a) is presented only in the range $\mathrm{r} / \mathrm{w} \in\{0-0.5\}$ for symmetry reason: indeed, the point $\left(\mathrm{r} / \mathrm{w}=0.5 ; \mathrm{Q}^{*}=0.5\right)$, common to all curves, corresponds to half of the input channel and the graph is symmetric around this point. As mentioned in the introduction, a major constraint is to design chips that can be considered filter-less, to avoid poisoning and clogging. We thus define a simple geometric criterion to delimit the filter-less situation: particle radius has to be inferior to one tenth of the minimal lateral channel dimension:

$$
\text { Filter-less criterion: } \quad r \leq 0.1 \min (h, w) .
$$


Figure 2(b) is a zoom of the same graph for values of $r / w \leq 0.1$, where we have also excluded portions of the curves that do not meet this criterion.

For any value of the aspect ratio, $\mathrm{Q}^{*}$ is an increasing function of $\mathrm{r} / \mathrm{w}$. It is quite obvious that for a fixed channel width w, the larger the layer of fluid considered, the larger the partial flow rate, so that $\mathrm{Q}^{*}$ increases with $\mathrm{r}$. More interestingly, this variation also means that for a given particle radius $\mathrm{r}$, a smaller channel width w corresponds to a higher partial flow ratio. If the fraction of fluid pumped in the sorted branch is an important criterion (which is for instance the case in applications involving expensive sample or sample available in low quantity), it is in general more efficient to work with smaller channels, keeping in mind that both $\mathrm{h}$ and $\mathrm{w}$ needs to be larger than 10r (filter-less situation).

Figure 2(b) and contour plot of $\mathrm{Q}^{*}$ in Figure 2(c) show that the maximum partial flow rate meeting the simple filter-less criterion of Equation 11 is obtained for an aspect ratio $\mathrm{h} / \mathrm{w}$ equal to 1 (square channel), and at the limit of the criterion: $r=0.1 h=0.1 \mathrm{w}$. However, the two following considerations lead to choose at least one of the two dimensions larger than this limit:

- technological limitation: since the objective is to sort submicrometric particles, $r / w=0.1$ corresponds to lateral dimension of order $1 \mu \mathrm{m}$, which is close to the resolution limit of most standard microfabrication techniques. A larger w permits a more robust fabrication process, with less influence of fabrication imperfections on the actual separation size.

- clogging issues: Clogging is a non-trivial phenomenon that depends on channel dimensions in a complex manner ${ }^{32-34}$. Equation 11 should thus rather be considered as a necessary condition, not as a sufficient one, to limit clogging. In addition, if the criterion is met exactly for the size of particles to be sorted, it is not met for bigger particles, present in the sample and that the device should exclude, so that the latter would be prone to clog the junction.

A larger cross section is also the most natural way to increase the flow rate: for applications where the flow rate ratio is not the relevant quantity, but extracted volume is, a compromise between small sorting size and larger dimensions should be considered.

Based on those elements, we describe in the next paragraphs two studies on designs corresponding to realistic situations, accessible to fabrication: 1) increased width for standard fabrication, relying on a two-level design; 2) high aspect ratio based on a 3D configuration enabled by a lamination-based process.

\section{Low aspect ratio: two-level output channel}

First, we determine geometries suited for a separation size of order $0.2-1 \mu \mathrm{m}$ (corresponding to critical sorting radius $r=100-500 \mathrm{~nm}$ ). The filter-less criterion leads to work with at least $\mathrm{h}_{0}=5 \mu \mathrm{m}$ high channels. Width at the intersection is set to $\mathrm{w}_{0}=50 \mu \mathrm{m}$, for the reasons already evoked (reduced clogging, fabrication accuracy, increased flowrate).

The length of main output $\mathrm{L}_{\text {out }}$ is fixed equal to $1 \mathrm{~mm}$, the minimum length needed between an intersection and an exit hole for standard microfabrication to keep a good design accuracy (punching of holes in PDMS or sand blasting in glass or silicon).

As first design, we consider the simple planar geometry depicted in Figure 3(a). We fix almost all the geometry according to the previous design and dimensions, the only unknown being the length of the lateral channel $\mathrm{L}_{\text {lat }}$ (sorted exit). $\mathrm{L}_{\text {lat }}$ is obtained thanks to our model by following the procedure described at the end of section 1. Figure 3(c) shows (red dotted line) the dependence of the length $L_{\text {lat }}$ of the lateral channel (width $\mathrm{w}_{\text {lat }}=5 \mu \mathrm{m}$ ) with the desired sorting radius $\mathrm{r}$. 
a)

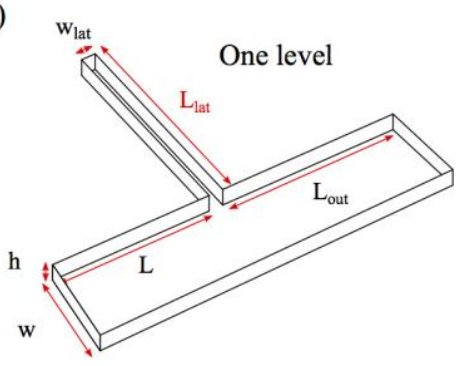

b)

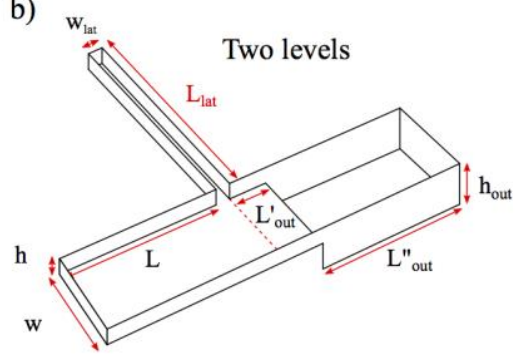

c)

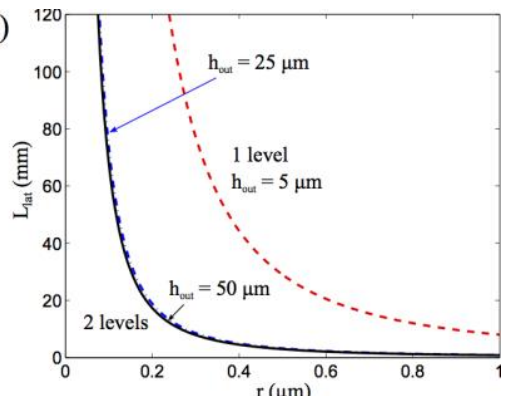

Figure 3 - Length of the lateral channel vs critical radius for two configurations. (a) Scheme of a simple, one-level device. (b) Two-level configurations designed to enable lower critical sorting size with shorter length of the secondary channel. (c) Computed length of the lateral channel vs critical radius for the two situations. Dotted red line corresponds to the design of Figure 3(a): one-level with widths $\mathrm{w}=50 \mu \mathrm{m}$ in input and output channels, wlat $=5 \mu \mathrm{m}$ in lateral channel, Lout $=1 \mathrm{~mm}$, and depth $\mathrm{h}=5 \mu \mathrm{m}$ for all channels. Blue dash-dotted line and solid black line correspond to the design of Figure 3(b) with two output depths. Input channel and lateral channel have the same geometry as previous case, and outlet channel has two-parts: first part with depth $\mathrm{h}=5 \mu \mathrm{m}$ and length $\mathrm{L}^{\prime}{ }_{\text {out }}=100 \mu \mathrm{m}$, second part with length $\mathrm{L}{ }^{\prime}{ }_{\text {out }}=1 \mathrm{~mm}$ and depth $\mathrm{h}_{\text {out }}=25 \mu \mathrm{m}$ (blue dash-dotted line) or $\mathrm{h}_{\text {out }}=50 \mu \mathrm{m}$ (solid black line).

The required length is already around $3 \mathrm{~cm}$ to sort $1 \mu \mathrm{m}$ diameter particles $(\mathrm{r}=0.5 \mu \mathrm{m})$, and becomes meter-long for submicron separation. This point constitutes a major drawback, on one hand for concrete integration in a microsystem (such channels have a large footprint, are difficult to realize without any defects with standard photolithography process) and on the other hand for practical use with reasonable separation time.

In order to overcome this issue, we propose to design a multilevel separator with an outlet channel much deeper than lateral channel. Indeed, for low aspect ratio, hydrodynamic resistance strongly depends on the depth (cubic dependence on the smallest dimension, $R_{h} \sim 1 / h^{3}$ ). Consequently, increasing the outlet depth drastically reduces the output resistance enabling much higher total flow rate and much lower lateral resistance. Therefore, we consider the case of two-level channels, as schematized in Figure 3(b). We keep 5- $\mu \mathrm{m}$ depth for a short part ( $\mathrm{L}_{\text {out }}, 100 \mu \mathrm{m}$ long) of the output channel just after the intersection, in order to ensure that the velocity profile is well described by Equation 1, so that we can relate the exit resistances to the sorting size by our model.

Similarly to the previous case, the value of critical radius is plotted as a function of lateral channel length (cross section $5 \times 5 \mu \mathrm{m}^{2}$ ) for two output channels depths: $\mathrm{h}_{\text {out }}=25 \mu \mathrm{m}$ and $50 \mu \mathrm{m}$. Figure 3(c) clearly shows that the two-level design enables a much lower length of the secondary channel needed in order to obtain sub-micron separation.

On a practical point of view, this design trick has strong advantages:

(i) It facilitates fabrication and integration: the strongly reduced lateral channel length limits risk of punctual defects of the photoresist, which become prohibitive for meter-long microchannels needed with one-level geometry, and thus enables design that is much more compact.

(ii) The filter-less approach makes our systems suitable for real samples: the lower dimension $(5 \mu \mathrm{m})$ is more than one order of magnitude larger than critical sorting size $(0.2-0.5 \mu \mathrm{m})$.

(iii) The typical velocity is higher and the residence time is strongly reduced. In the case of $h_{\text {out }}=h=5$ $\mu \mathrm{m}$ (one-level situation), for a system designed with a critical sorting size $0.45 \mu \mathrm{m}\left(\mathrm{r}_{\mathrm{c}}=0.225 \mu \mathrm{m}\right)$, a particle will need on the average $\sim 40$ minutes to travel from the inlet to the lateral outlet (total pressure difference from the inlet to the two outlets set to 1 Bar). This time drops to a few minutes for two-level designs: approximately 3.9 and 3.5 minutes for $h_{\text {out }}$ values of 25 and $50 \mu \mathrm{m}$ respectively. This gain of almost two orders of magnitude permits to extract the sorted particles within minutes, a reasonable sample preparation time if the block is envisioned in an integrated sensor. In this particular design, the residence time decreases only slightly when $h_{\text {out }}$ increases from 25 to $50 \mu \mathrm{m}$ in spite of the strong height-dependence of the resistance, mostly because of the contribution of the narrow part of the outlet channel to the resistance (right after the branching point, length $\mathrm{L}^{\prime}{ }_{\text {out }}=100 \mu \mathrm{m}$, depth $\mathrm{h}_{0}=5$ $\mu \mathrm{m})$. 
The validity of those results, obtained from a simplified analytical approach, is confirmed both experimentally and numerically as will be discussed in section E.

\section{High aspect ratio: 3D configuration}

As already presented, real design should move aside from a square channel at the filter-less limit $(\mathrm{h}=$ $\mathrm{w}=10 \mathrm{r}$ ), to limit issues related to clogging, because of fabrication accuracy, and to increase the flowrate. The previous situation, increasing the width $\mathrm{w}$, corresponds to the most directly accessible design: the fabrication of high aspect ratio structures is very delicate for usual techniques (soft lithography and molding of PDMS, or etching of silicon). However this is not necessarily the best option: the contour plot of Figure 2(c) shows that for a fixed particle dimension (fixed r), the flow rate ratio is much more affected by $w$ than $h$ : iso-values of $\mathrm{Q}^{*}$ are almost vertical on the $(\mathrm{r} / \mathrm{h}, \mathrm{r} / \mathrm{w})$ representation of Figure 2(c). In other words, efficiency (or flow rate ratio) would be much less degraded by keeping $w$ small and increasing $h$.

To overcome this limitation, we take advantage of an alternative 3-dimensional configuration depicted in Figure 4(a), which enables pumping fluid in the direction orthogonal to the main channels plane. To that purpose, we use a multi-layer lamination-based technique that we have developed initially with SU- $8^{35}$ and extended recently to other dry films. ${ }^{36}$ A SEM micrograph of the system is shown in Figure 4(b), fabrication details are given in section D.

a)

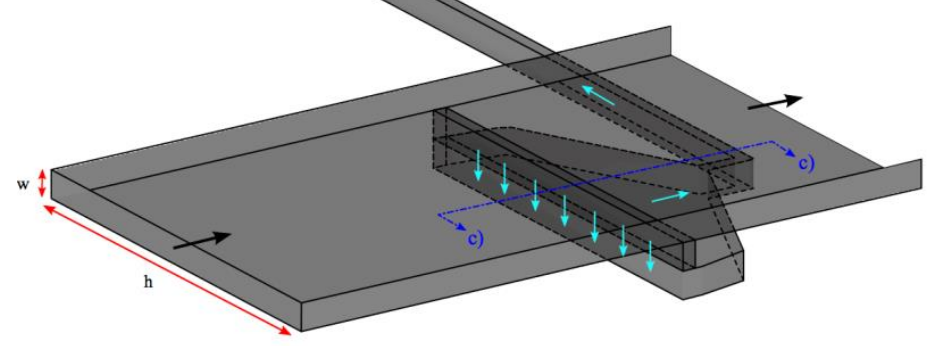

b)

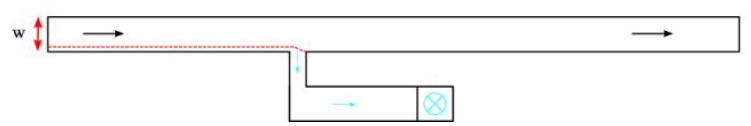

c)

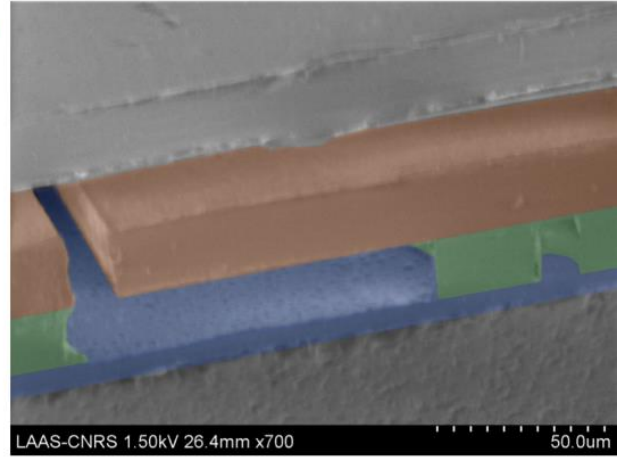

Figure 4- Design of 3D configuration. (a) 3D scheme of the channel architecture. (b) 2D cross section of the same design. (c) SEM image of a chip (for clarity, different artificial colors have been added, one for each laminated layer).

Briefly, successive lamination of several layers that can each be patterned by photolithography enables superimposing a main channel (corresponding to the in and out branches of Figure 1) to a secondary channel (corresponding to the lateral branch). This configuration extracts fluid in the "vertical" direction instead of the planar one, and thus reverses the aspect ratio issue.

Based on this approach, we can design a chip with lateral dimension $\mathrm{h}=300 \mu \mathrm{m}$ (notations are chosen to be consistent with the ones used in the model) and thickness $\mathrm{w}=20 \mu \mathrm{m}$, which corresponds to an aspect ratio $\mathrm{h} / \mathrm{w}=15$. A sorting size equal to $1.3 \mu \mathrm{m}$ can be obtained with a secondary channel of length $\mathrm{L}_{\text {lat }}=30 \mathrm{~mm}$ ( with $\mathrm{h}_{\text {lat }}=\mathrm{w}_{\text {lat }}=20 \mu \mathrm{m}$ ), extracting particles to another layer (see Figure 4(a)).

This configuration is a good compromise between sample volume and efficiency. As a matter of fact, its large cross-section (h.w) enables substantial flow rate with moderate pressure drop (in the sorted output, $\mathrm{Q}_{\text {lat }}=0.6 \mu \mathrm{L} / \mathrm{min}$ with $\Delta \mathrm{P}=1 \mathrm{Bar}$ ), with a quite small sorting size, while the partial flow rate $\mathrm{Q} *$ is kept superior to $0.5 \%$. 


\section{Materials and methods}

\section{Microsystems fabrication}

Two level channels are realized following two technological routes: either in polydimethylsiloxane (PDMS) by standard polymer soft-lithography ${ }^{37}$, or in silicon/glass by dry etching of silicon (deep reactive ion etching, DRIE) and anodic bonding.

For PDMS process, we choose SU-8, a negative photoresist, as the structural material for the two-level mold. A first $5 \mu \mathrm{m}$ thick layer of SU-8 is spin-coated and soft-baked, and finally UV exposed with a MA150 mask aligner (Suss Microtec). The first layer is then baked and uncrosslinked SU-8 is developed in PGMEA. Hard bake at $120^{\circ} \mathrm{C}$ is used to complete the reticulation of this first layer. A second $20 \mu \mathrm{m}$ thick SU-8 layer is then spin coated and the steps already described for layer one are repeated. An octadecyltrichlorosilane layer is then deposed on the mold by 2 minutes incubation of $1 \%$ solution in xylene, as an anti-adhesive treatment. A 10:1 weight mixture of PDMS/curing agent (Sylgard 184, Dow Corning) is then casted on the mold and baked ( 2 hours, $65^{\circ} \mathrm{C}$ ). It is then pealed off from the mold, cut to microscope cover glass size and washed in a bath of isopropanol. PDMS and glass microscope coverslip are plasma sealed.

The silicon/glass process is performed using 4-inch wafers. The silicon wafer is first cleaned using $\mathrm{O}_{2}$ plasma and piranha solution (equal volumes of $\mathrm{H}_{2} \mathrm{SO}_{4}$ and $\mathrm{H}_{2} \mathrm{O}_{2}$ at $20 \%$ in water). The first level of channel $(5 \mu \mathrm{m}$ depth) is then etched in the silicon wafer by photolithography (AZ ECI 3012, Microchemicals) followed by reactive ion etching with $\mathrm{SF}_{6}$ and $\mathrm{C}_{4} \mathrm{H}_{8}$ plasma (process parameters: flow rates $\mathrm{SF}_{6}=200 \mathrm{sccm}$ and $\mathrm{C}_{4} \mathrm{~F}_{8}=400 \mathrm{sccm}, \mathrm{P}=0.07 \mathrm{mbar}$, power of the source generating the plasma $\mathrm{P}_{\text {icp }}=2800 \mathrm{~W}$, power of the accelerating source $\mathrm{P}_{\text {bias }}=60 \mathrm{~W}, \mathrm{t}=7 \mathrm{~min}$ ). The second level of channels (50 $\mu \mathrm{m}$ depth) is obtained with a DRIE process operated in a pulsed manner by repeating three steps of etching, protection, and surface activation with the following process parameters: step (1) $\mathrm{SF}_{6}=700 \mathrm{sccm}, \mathrm{P}=0.08 \mathrm{mbar}, \mathrm{P}_{\text {icp }}=2300 \mathrm{~W}, \mathrm{P}_{\text {bias }}=85 \mathrm{~W}$ for $\mathrm{t}=6 \mathrm{~s}$; step (2) $\mathrm{C}_{4} \mathrm{~F}_{8}=350 \mathrm{sccm}, \mathrm{P}=0.07 \mathrm{mbar}$, $\mathrm{P}_{\text {icp }}=2800 \mathrm{~W}, \mathrm{P}_{\text {bias }}=90 \mathrm{~W}$ for $\mathrm{t}=3.5 \mathrm{~s}$; step (3) $\mathrm{O}_{2}=200 \mathrm{sccm}, \mathrm{P}=0.06 \mathrm{mbar}, \mathrm{P}_{\text {icp }}=2800 \mathrm{~W}, \mathrm{P}_{\text {bias }}=90 \mathrm{~W}$, $\mathrm{t}=10 \mathrm{~s}$ ) during $6 \mathrm{~min}$.

Fluid-access holes are drilled in the silicon wafer using a powder blasting system, after deposition of a film of dry resist to protect the channels. Finally, the Si and glass wafers are aligned and bonded using a standard anodic bonding process $\left(600 \mathrm{~V}\right.$ at $370^{\circ} \mathrm{C}$ for $\left.10 \mathrm{~min}\right)$.

Avoiding dusts and defects in the photoresist during photolithography is quite critical in the process, because the total surface covered by each channel is of order $1 \mathrm{~mm}^{2}$ for each chip. However, this is acceptable thanks to two-level design. With one-level channel, meter-long lateral channel would cover around $1 \mathrm{~cm}^{2}$, and obtaining zero defects on the whole surface would have been extremely demanding in term of microfabrication.

The two-level trick allows compact design, an example of which is shown in Figure 5(a-b). On a 4inch wafer, we realize twenty-five designs with six different critical sizes. 
a)

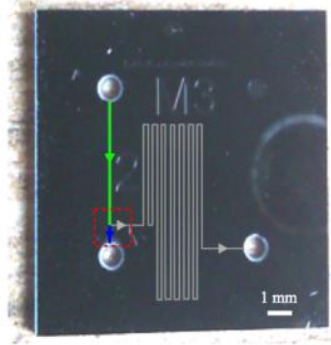

c)

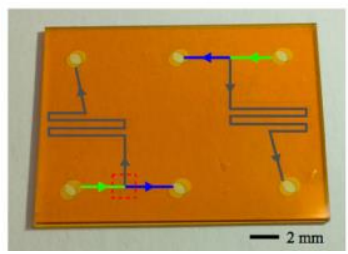

b)

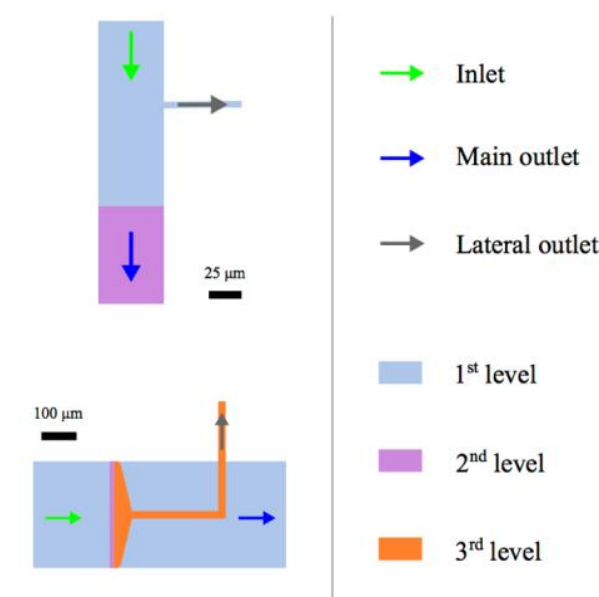

Figure 5- Fabricated chips and associated mask layouts for the intersecting part. (a-b) Two-level geometry described above (glass-silicon technology), (c-d) Dry film technology (3D configuration).

The position where the main output channel becomes deeper is not aligned with the intersection, but is shifted at $100 \mu \mathrm{m}$ downstream, which ensures that the velocity profile at the intersection is the one of Equation 1. This shift contributes to the total value of the output hydrodynamic resistance, for about $25 \%$ in the case of $25 \mu \mathrm{m}$ deep channels (PDMS devices), and more than $50 \%$ for $50 \mu \mathrm{m}$ ones (glass/silicon chips).

Three dimensional systems fabrication is based on development work that was previously described ${ }^{36}$. Substrate (4", $500 \mu \mathrm{m}$ Schott AF32 glass wafer - to allow visualization with inverted microscope) is cleaned with $\mathrm{O}_{2}$ plasma $(800 \mathrm{~W}, 15 \mathrm{~min})$ and piranha solution $(2 \mathrm{~min})$. A first layer $(5 \mu \mathrm{m})$ of dry film photoresist (DF-1005, EMS) is laminated on substrate (Shipley laminator, 2.5 bars, $100^{\circ} \mathrm{C}, 1 \mathrm{~m} / \mathrm{min}$ ), and then UV exposed and baked (this level is not structured). Four levels of dry films $(20 \mu \mathrm{m}, \mathrm{DF}$ 1020 , EMS ) are then laminated $\left(2 \mathrm{bars}, 65^{\circ} \mathrm{C}, 0.5 \mathrm{~m} / \mathrm{min}\right)$, and exposed. A hot plate with ramp of temperature is used for post exposure bake, and photoresist is developed with cyclohexanone (2 to 5 mins). Before laminating each additional layer, a surface treatment $\left(\mathrm{O}_{2}\right.$ plasma, 200W, $\left.2 \mathrm{~min}\right)$ is applied to improve surface adhesion between the dry films. At the end of the process, wafer is cut with diamond saw and 14 microfluidic chips are released, a chip containing two devices is shown in Figure $5(\mathrm{c}-\mathrm{d})$. Fluid inlets and outlets are made out of the film material; the glass substrate is exclusively used for mechanical support.

\section{Microfluidic experiments}

Fluorescent calibrated polystyrene microbeads with diameter 0.1 to $2 \mu \mathrm{m}$ (FluoSpheres ${ }^{\circledR}$, Invitrogen) are used as fluorescent tracers, with excitation/emission wavelengths centered at 505/515 nm and $580 / 605 \mathrm{~nm}$ for green and red spheres respectively. Nominal particles sizes were confirmed by dynamic light scattering with a commercial setup (Zeta sizer Nano ZS, Malvern).

Commercial solutions are diluted in deionized water to obtain typical working concentration in the range $0.01 \%$ in volume, to avoid particle-particle interactions.

Observations are done using an inverted fluorescence microscope (Zeiss, Axio Observer D1) equipped with $x 100$ oil immersion objective (numerical aperture NA $=1.3$ ). Images are recorded with an EMCCD camera (Andor iXon) and data processing is performed using Image J (open source, NIH).

Flows are imposed with a pressure controller (Fluigent MFCS); tubings are connected directly to the PDMS chips. For Glass/Silicon and Dry film chips, we use an in-house chip holder (aluminium base, peek or Teflon manifold, the latter making the connection between the chip with a standard o-ring and a ferrule for Teflon tubing).

We observe the flow at the branching point, which enables us to experimentally determine, for each suspension, whether the critical sorting size of the device is lower or higher than the diameter of the particles. For each setup, we vary the imposed pressure from $100 \mathrm{mBar}$ to 1 Bar. As an example, 
Figure 6 shows $0.5 \mu \mathrm{m}$ (a) and $0.1 \mu \mathrm{m}$ (b) beads flowing in a $0.32 \mu \mathrm{m}$ critical diameter at an imposed pressure of $800 \mathrm{mBar}$.

The same visualization methodology is used for three dimensional chips (using $1 \mu \mathrm{m}$ green fluorescent and $2 \mu \mathrm{m}$ red fluorescent beads), and the separation of the $1 \mu \mathrm{m}$ particles ( $2 \mu \mathrm{m}$ ones remaining in the main flow) is observed.

\section{Numerical methods}

In order to discern the limits of our analytical model, based on three hydraulic resistances (Figure 1(c)), we use a Finite Element Method (F.E.M.). Stokes equation is solved with COMSOL Multiphysics for an incompressible Newtonian fluid (water) in a 3D system composed of two rectangular channels, forming a T-junction, and based on the geometry described in C.1. (Figure 3(a)).

The lengths of the main channel $(\mathrm{w}=50 \mu \mathrm{m}, \mathrm{h}=5 \mu \mathrm{m})$ and side channel (wlat $=5 \mu \mathrm{m}, \mathrm{h}=5 \mu \mathrm{m})$ are set arbitrarily to $200 \mu \mathrm{m}$ and $50 \mu \mathrm{m}$ in order to reduce computational time. It is checked that the flow is fully established at the inlet and outlets locations. Given the reflection symmetry centered at the $\mathrm{z}=$ $\mathrm{h} / 2$ plan, simulation is carried out for only half of the geometry $(\mathrm{z}=2.5$ to $\mathrm{z}=5 \mu \mathrm{m})$.

A mapped mesh is applied, with cells reduced in size when close to the walls and near the junction (geometric progression), where a high resolution on velocity profile is required. Cell sizes range from about $15 \mathrm{~nm}$ to $8.5 \mu \mathrm{m}$, and total number of elements reached 278000 .

We impose constant pressure as the three boundary conditions. We calculate the correct values at the inlet and outlets of the simulated T-shaped geometry from the pressure difference between the inlet and the two outlets of the chip that is set to 1 bar. To this end, we use a theoretical model of hydraulic resistance and the channel lengths of the chip designed for a critical radius $r=250 \mathrm{~nm}$. An iterative solver (generalized minimal residual method) is applied to find the pressure and velocity fields. The inlet flow rate obtained numerically is consistent within $1 \%$ with the one calculated from Equation (2).

One of the goals of this numerical approach is to determine which region of the inlet channel flows into the lateral channel. In other words, we need to localize where the flow rate in the lateral outlet is originating from. One way to look at this information is to calculate the flow streamlines. We screen origin positions of streamlines at the inlet, and we determine whether they are flowing in the lateral channel. It enables to obtain the profile of the separation curve, i.e. the simulated critical radius that depends on $\mathrm{z}$. This profile is fitted with a third degree polynomial equation.

To check the precision of this method, we compare the flow rate passing through the section defined by the separation curve to the flow rate in the lateral outlet. Flow rates are calculated by integrating the velocity (values from F.E.M. solving) over the area of interest (at the inlet, between the side of the channel and the curve of the critical radius - gray area on Figure 8(a) -, and over the lateral outlet section). A relative error of less than $1 \%$ is obtained between those flow rates, validating this method to establish the critical radius profile.

\section{E. Results and discussions}

For the 2D configuration, Figure 6(d) summarizes the experimental results, where particles with diameter $0.1-1 \mu \mathrm{m}$ are injected in separators with predicted critical diameter $0.15-1.2 \mu \mathrm{m}$. Those microsystems are designed following the procedure described in Section B, with the low-aspect ratio, two-level design explicated in Section C-1. 

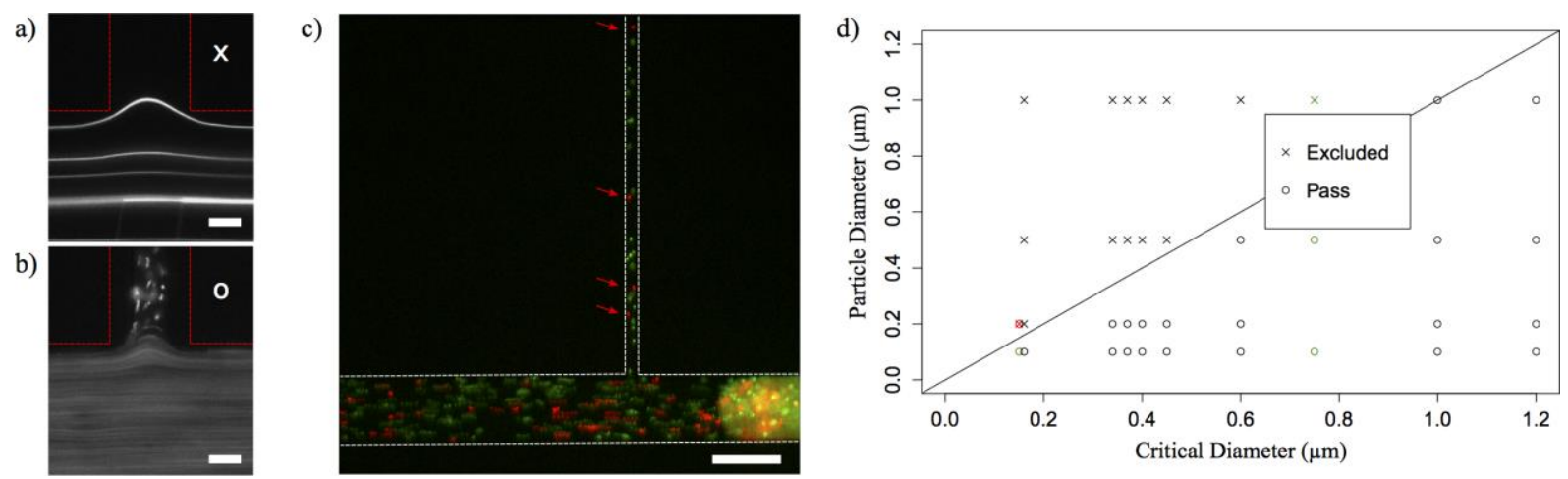

Figure 6- Experimental results. (a-b) Micrographs obtained at a $0.32 \mu \mathrm{m}$ critical size branching, with particles injected from left to right with a $800 \mathrm{mBar}$ imposed pressure: (a) $0.5 \mu \mathrm{m}$ diameter particles. (b) $0.1 \mu \mathrm{m}$ diameter particles. Scale bar is $2 \mu \mathrm{m}$. Red dashed lines show the position of channels' walls. (c) Results with beads mixtures. Simultaneous flow of $200 \mathrm{~nm}$ red particles and $100 \mathrm{~nm}$ green particles, injected at equal concentration $\left(4.510^{9}\right.$ particles per $\mathrm{mL}$ ) inside a branching designed for a critical diameter equal to $0.15 \mu \mathrm{m}$. Scale bar is $50 \mu \mathrm{m}$. (d) Summary of experimental results: each point on the graph represents a set of experiments where a suspension containing particles of a certain size is injected in a separator and the pressure ranges from $100 \mathrm{mBar}$ to $900 \mathrm{mBar}$. Horizontal axis represents the critical predicted size of the separator, and vertical axis is the diameter of the injected particles. If during an experiment, particles flow through the side channel, the experiment is represented as an o. If no particle is entering the side channel, the experiment is represented as an $\mathrm{x}$. The solid line represents the theoretical limit determined from the model presented in Sections B-C. Symbols represented in color (green and red) correspond to experiments where beads of different sizes are injected simultaneously.

The size of the particle is presented on the vertical axis, and the predicted theoretical critical size of the system on the horizontal one. The solid line represents the theoretical separation, ie, a particle above this line should not flow through the lateral channel. Particles flowing through are presented with a circle and particles that do not with an X. For most experiments, realized with only one type of particles (represented with black symbols), experimental results are in quantitative agreement with predicted sorting size. $0.2 \mu \mathrm{m}$ diameter particle do not flow into the secondary channel of the $0.16 \mu \mathrm{m}$ separator. We can therefore conclude we achieved separation down to $200 \mathrm{~nm}$. In addition, each point on the graph represents a set of data with different pressure drops applied ranging from 100 to $900 \mathrm{mBar}$, and we did not notice any changes when increasing or decreasing the pressure.

We also realized experiments in the 2D configuration with mixtures of beads with two different sizes, which constitutes a step further toward real systems. For a $0.75 \mu \mathrm{m}$ separator, $1 \mu \mathrm{m}$ diameter beads were excluded from the lateral channel, when flowing simultaneously either with $100 \mathrm{~nm}$ or with 500 $\mathrm{nm}$ beads, consistently with predictions (green symbols at critical diameter $0.75 \mu \mathrm{m}$ in Figure 6(d)). It corresponds to an efficiency $Q^{*}$ around $0.1 \%$. For a $0.15 \mu \mathrm{m}$ separator, we tried to exclude $200 \mathrm{~nm}$ particles (red fluorescent label), flowing with $100 \mathrm{~nm}$ particles (green fluorescent label). In that particular case, the exclusion of beads is only partial, even though the number of $200 \mathrm{~nm}$ particles flowing in the lateral channel corresponds to about ten-time dilution of the sample. Indeed, given the initial beads concentrations ( $4.510^{9}$ beads. $\mathrm{mL}^{-1}$ for both sizes), there would be about 45 beads of each type in the $10^{-8} \mathrm{~mL}$ of the visible part of the lateral channel, and we observe only 4 of the $200 \mathrm{~nm}$ ones (red arrows in Figure 6(c)). This non-perfect exclusion of $200 \mathrm{~nm}$ beads, together with the apparent discrepancy with the result presented above on a similar device (perfect exclusion of $200 \mathrm{~nm}$ beads for $0.16 \mu \mathrm{m}$ separator), can first be attributed to uncertainty in channels dimensions, related to the technological process. A lateral channel slightly larger or deeper than designed can lead to an error in the sorting size. A rough estimate, based on photolithography resolution and on accuracy of characterization of microchannel depth leads to around 10 percent. Indeed, depth variations measured by mechanical profilometry are $\pm 0.2 \mu \mathrm{m}$ for the 5 - $\mu \mathrm{m}$ deep channels (homogeneity on a given wafer, as well as reproducibility of the etching process from one batch to the other). This can account for the discrepancy between the perfect exclusion of the $200 \mathrm{~nm}$ beads observed for the $0.16 \mu \mathrm{m}$ separator whereas the $0.15 \mu \mathrm{m}$ separator only partly works. 
However, additional arguments are needed to explain the partial exclusion: particles Brownian motion, and actual shape of the separating velocity streamline. We discuss in the last part of the article how they affect the performances, after presenting results obtained with $3 \mathrm{D}$ devices.

Let us discuss sample purity at the different exits. Yamada et al. expressed it in their original demonstration of hydrodynamic filtration as the ratio of large vs. small particles at each outlet, and compared these ratios with the one at the inlet, which defines sample purification for a chosen set of sizes. Our work is focused on the extraction of small sizes, particles concentration is not aimed at, and in most situations particles are introduced separately. We thus focus on the ratio of output vs. input concentration for each particle size, which could be viewed as collection efficiency as function of size.

In the main, non-sorted outlet, the ratio of output vs. input concentrations is equal to 1 for all particle sizes: the composition of the solution is very similar to the injected one and no purification of the sample is achieved.

In the lateral channel, this number is close to unity for small particles (smaller than the sorting diameter), showing no dilution or concentration effect, and is strictly equal to zero for particles larger than the sorting size, since they are excluded for a large majority of our experimental results (crosses above the solid line in Figure 6(d)). The only chip for which it is not the case is the $0.15 \mu \mathrm{m}$ separator that has already been described. Even for this non-perfect case, purification of $100 \mathrm{~nm}$ vs. $200 \mathrm{~nm}$ beads is around ten-fold: small particles are kept at their initial concentration as expected whereas 200 $\mathrm{nm}$ beads, slightly larger than the sorting size, are diluted 10 times. For all the other results presented in Figure 6(d), showing complete exclusion of large particles, the purification of small particle is ideal.

In summary, the device does not purify large particles (the solution exiting in the main output does not differ much from the input solution), but it enables to extract in the sorted outlet a purified, not concentrated, solution of small particles.

a)

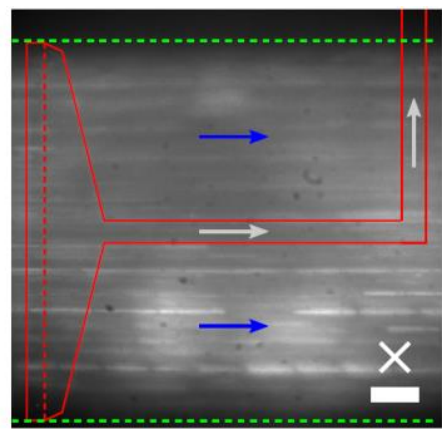

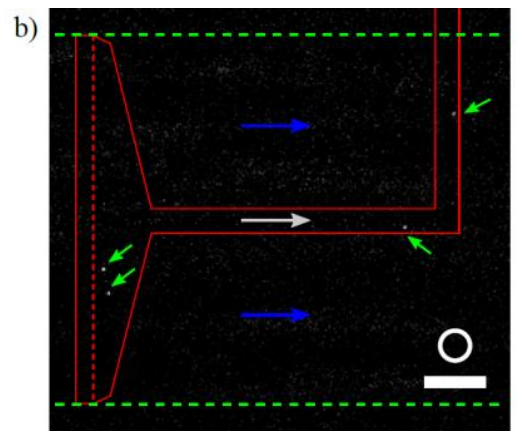

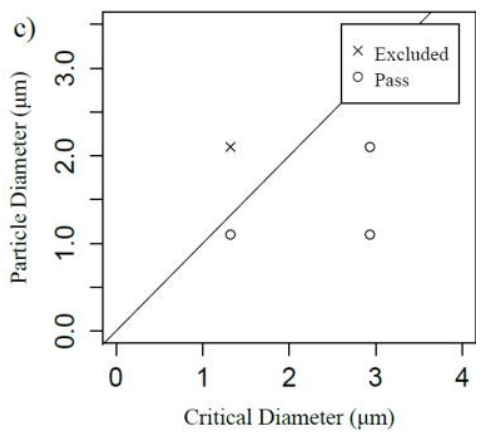

Figure 7- Experimental results obtained with the 3D device. (a-b) Micrographs of exclusion or aspiration of 1 or $2 \mu \mathrm{m}$ diameter beads for a $1.3 \mu \mathrm{m}$ critical diameter. Scale bars are $50 \mu \mathrm{m}$. Pressure difference between inlet and outlets is $10 \mathrm{mbars}$. The particles are flowing from left to right: arrows symbolize the flow in the main outlet channel (blue arrows) and in the lateral channel (light grey arrows). (a) $2 \mu \mathrm{m}$ (red fluorescent) particles are flowing in the main channel and do not enter in the lateral channel. (b) $1 \mu \mathrm{m}$ (green fluorescent) particles flow in both main and lateral channels (green arrows point at particles). (c) Summary of experimental results for two sizes of beads, obtained with one device operated with different output/lateral pressures enabling to tune the partial flow rate $\mathrm{Q}^{*}$ (see Equation 4), and thus the critical diameter. Horizontal axis shows the critical diameter $\mathrm{d}_{\mathrm{c}}$ of the device for specific applied pressures: $\mathrm{d}_{\mathrm{c}}=1.3 \mu \mathrm{m}$ for $\Delta \mathrm{P}^{*}=1$ and $\mathrm{d}_{\mathrm{c}}=2.9 \mu \mathrm{m}$ for $\Delta \mathrm{P}^{*}=1 / 3$. If during an experiment, particles flow through the side channel, the experiment is represented as an o. If no particle is entering the side channel, the experiment is represented as an $\mathrm{x}$.

For the 3D configuration, with a chip designed to exclude particles above $1.3 \mu \mathrm{m}$, we obtain similar behavior: the exclusion of $2 \mu \mathrm{m}$ diameter beads, whereas $1 \mu \mathrm{m}$ beads flow through the lateral one, as shown in Figure 7(a-b). It is also possible, with a single device, to test different critical diameters conditions when adjusting the ratio of pressure differences $\Delta \mathrm{P}^{*}$ (see Equation 4, Part B). Setting different pressures at the main and lateral outlets ( $P_{\text {in }}=510$ mbars, $P_{\text {out }}=500$ mbars, $P_{\text {lat }}=480$ mbars $)$, corresponding to $\Delta \mathrm{P}^{*}=1 / 3$, allowed us to obtain a critical diameter of $2.9 \mu \mathrm{m}$, and we indeed observed that in these conditions the two sizes of particles ( 1 and $2 \mu \mathrm{m}$ diameter) are drained through the lateral channel, as expected (see Figure 7.c). 
When applying a pressure difference of 1 bar, samples can be processed within a few minutes. Thanks to this geometry, resistances are small, and we achieve high flowrates $\mathrm{Q}_{\text {in }} \sim 180 \mu \mathrm{L} / \mathrm{min}, \mathrm{Q}_{\text {lat }} \sim 0.6$ $\mu \mathrm{L} / \mathrm{min}$. Travel times are consequently very short: $\sim 1.2 \mathrm{~s}$ for a particle to flow between the inlet and the lateral outlet, and in the order of $10 \mathrm{~ms}$ if only flowing in the main channels (microscope observation is in that case restricted to the lateral channel and the inlet/outlet).

It is also worth mentioning that while silicon/glass or PDMS devices can withstand rather high pressures (>10-15 bars), dry films chips are limited to only 4-5 bars before delamination. As a result, when higher flowrate is needed, and whereas the standard response would be to increase the pressure difference for a silicon-based system, the geometry (lower hydraulic resistances) becomes an important parameter for our laminated chips.

As evoked in the presentation of results concerning 2D devices, we now discuss the two phenomena that can cause the microfluidic chip to depart from the ideal behavior described: (i) the Brownian motion of the particles, which plays a strongest role for the smallest particles, and (ii) the real shape of the separating streamline.

\section{(i) Influence of particle diffusion on sorting size}

We estimate the error on critical radius $r$ due to diffusion by the following simple argument. A particle of radius equal to $r+\Delta r$ should be excluded from the lateral channel. However, it can enter if, during its passage in front of the bifurcation, its Brownian motion is sufficient to displace its center of mass to the streamlines entering the lateral channel. The time spent by the particle in front of the junction can be estimated as $t_{\text {pass }}=\frac{w_{\text {lat }}}{v_{r}}$, where $w_{\text {lat }}$ is the width of the lateral channel and $v_{r}$ is the velocity in the main channel at a distance $r$ of the wall. Since $r$ is much smaller than the width and height, we can consider that the velocity profile is linear within the zone located between the wall and $\mathrm{r}$, so that $v_{r} \sim \dot{\gamma}_{w} r$, where $\dot{\gamma}_{w}$ is the shear rate at the vertical wall (located at $\mathrm{y}=-\mathrm{w} / 2$ with the notations of Figure 1). $\dot{\gamma}_{w}$ can be assumed equal to the shear rate on the top wall, which for the low aspect ratio considered here (parabolic velocity profile in the $\mathrm{z}$ direction) reads $\dot{\gamma_{w}}=\frac{\Delta P h}{2 \eta L}$ where $\mathrm{L}$ is the length on which the pressure difference is applied. We thus obtain $v_{r} \sim \frac{\Delta P h}{2 \eta L} r$. The typical distance explored by Brownian motion is $\Delta r=\sqrt{2 D t_{\text {pass }}}$.

With the Stokes-Einstein formula $D=\frac{k T}{6 \pi \eta r}$, it finally leads to:

$$
\Delta r \sim \sqrt{\frac{2 k T}{3 \pi r^{2}} \frac{w_{\text {lat }} L}{\Delta P h}} .
$$

The process being due to a competition between convection and diffusion, we can also express the error with a dimensionless, Peclet number. We thus define $P e_{\text {pass }}=\frac{t_{\text {diff }}}{t_{\text {pass }}}$ as the ratio of diffusion to convection times, considering the length scales actually involved: diffusion over a distance equal to the particle size, and convection over a distance $w_{\text {lat }}$ at a velocity $v_{r}$. It reads: $P e_{\text {pass }}=\frac{r^{2}}{D} / \frac{w_{\text {lat }}}{v_{r}}$.

Combining with the previous equations, we obtain for the relative blurring: $\frac{\Delta r}{r} \sim\left[P e_{\text {pass }}\right]^{-\frac{1}{2}}$, showing that the effect is indeed fully controlled by $P e_{\text {pass }}$.

For $\mathrm{r}=150 \mathrm{~nm}$, the estimated error due to particle diffusion is comparable to particle size: $\Delta r \sim 60 \mathrm{~nm}$ for $\Delta P=1$ bar, and $\Delta r \sim 200 \mathrm{~nm}$ for $\Delta P=100 \mathrm{mBar}$.

Equation 12 shows that the sorting process of hydrodynamic filtration is more affected by diffusion for slow flows (the main velocity being controlled by $\Delta P, \mathrm{~L}$ and $\mathrm{h}$ ), since it is related to a competition between diffusion vs convection.

We can note that $\Delta r$ is independent on the viscosity of the solution, because of a compensation of its effect on convection (flow velocity) and diffusion (particle Brownian motion). 
The most important point that Equation 12 evidences is the strong dependence on particle radius, as $1 / r$. For realistic pressures and dimensions, this effect becomes prohibitive for sorting particles in the 10 -nanometer range, whereas it is completely negligible for micron-sized objects. Indeed, for $\mathrm{r}=1$ $\mu \mathrm{m}$, the error is only $10 \mathrm{~nm}$ for $\Delta P=1 \mathrm{Bar}$, lower than half percent when compared to the particle size.

Our study in the 0.1-1 $\mu \mathrm{m}$ range thus corresponds to the limit in small dimensions where the approach can be considered relevant. For the typical sizes aimed in this work $(\mathrm{r}=250 \mathrm{~nm}), \Delta r \sim 40 \mathrm{~nm}$ for $\Delta P$ $=1 \mathrm{bar}$, and $\Delta r \sim 120 \mathrm{~nm}$ for $\Delta P=100 \mathrm{mBar}$.

In summary, diffusion can significantly contribute to blurring the exact sorting limit, but remains acceptable for sufficiently fast flows, down to hundred-nanometer sized objects.

(ii) Influence of the real velocity field: local hydraulic resistance and shape of the separating streamline

The theoretical model developed in section $\mathrm{B}$ to determine the flow ratio $Q^{*}$ and the critical radius is based on two assumptions which are discussed now. To this end, we use results from the numerical simulation presented in the section D.3. First, the local pressure drop caused by the bifurcation was neglected. If we compare the value of $Q^{*}$ obtained by simulation with the one deduced from the analytical model, the relative error is $-5.8 \%$. It can be explained in part by the non-Poiseuille flow, with the presence of a recirculating flow at the entrance of the lateral channel (see the streamlines on Figure 8(c)).

a)

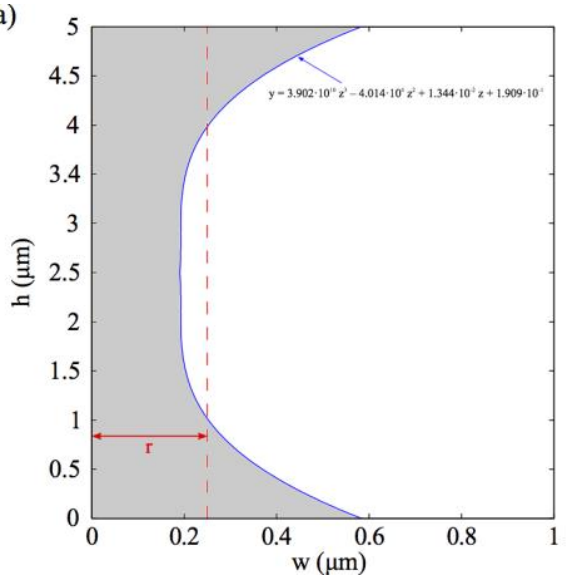

b)

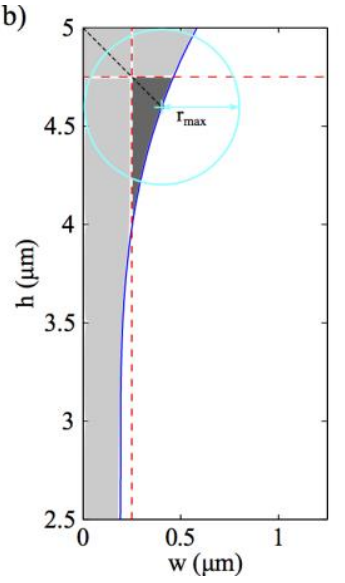

c)

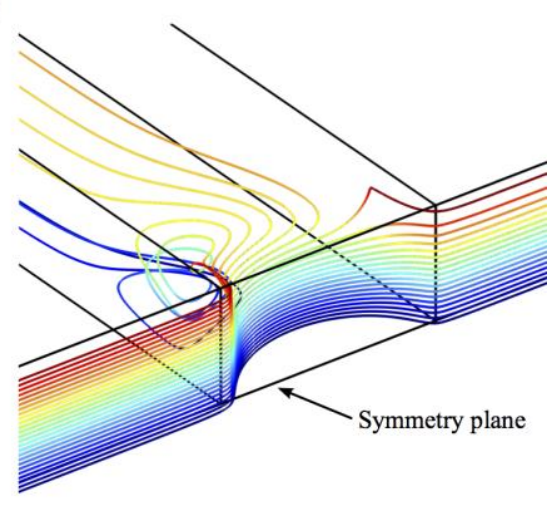

Figure 8- Numerical results: red dashed line represents the theoretical critical radius (a) evolution of $r_{c}$ along the $z-$ axis. (b) Determination of $r_{\max }$. The dark grey zone shows the region in which particles with a radius bigger than the theoretical critical radius can flow into the lateral channel. (c) Streamlines in the separating zone originating from the input channel at a distance from the wall corresponding to the predicted critical radius $\left(\mathbf{y}=\mathbf{w} / \mathbf{2}-\mathbf{r}_{\mathbf{c}}\right)$. Colors are coding for the altitude $(\mathrm{z})$. Only the superior half of the channel is displayed since the mid-plane $\mathrm{z}=\mathrm{h} / 2$ is a symmetry plane.

Second, the critical radius is considered constant all along the z-axis direction. This assumption would be true in the case of a channel with an infinite height. In our case, all the streamlines which go inside the lateral channel do not belong to the same vertical plane, because of the influence of the top and bottom walls. Figure 8(a) represents the profile obtained by simulation. It shows that the simulated critical radius is slightly lower than the theoretical one (red dashed line) at mid-height $(\mathrm{z} \sim 2.5 \mu \mathrm{m})$ and becomes higher close to the top and bottom walls. The maximum critical radius $\left(\mathrm{r}_{\max }\right)$ is fixed by the intersection between the separation profile and the expression $\mathrm{z}=\mathrm{h}-\mathrm{y}$, which corresponds to the biggest particle that can have its center located inside the separated fluid (see on Figure 8(b)). $r_{\max }$ is equal to $403 \mathrm{~nm}$, almost twice the theoretical radius $(250 \mathrm{~nm})$. The flow going to the lateral channel and including particles with a radius bigger than the theoretical one is computed by numerical integration of the velocity (dark grey zone on Figure 8(b). This flow is equal to $16 \%$ of $Q_{\text {lat }}$, meaning that almost one sixth of the fluid going to the lateral channel is originating from a region that should 
be excluded (since it is located beyond the critical radius value). This phenomenon offers one explanation to why particles bigger than the critical dimensions can pass through the lateral channel.

\section{F. Conclusions}

We demonstrate the sorting of submicrometric particles by hydrodynamic filtration. The model we develop enables predicting filtration performances for any aspect ratio of the input channel. We propose a two-level geometry to realize sorting in short times (a few minutes), and a 3D configuration based on lamination to achieve higher flow rates (of order $\mu \mathrm{L} / \mathrm{min}$ ). We confirm the validity of our model both experimentally by systematic investigation of the performances of fabricated systems, and numerically by resolving the actual shape of the separation streamlines envelop. We also assess the limit of the approach due to Brownian diffusion.

We achieve separation down to $200 \mathrm{~nm}$, which is to our knowledge the lowest size obtained with a passive, filter-less device, purely based on hydrodynamics.

This work also constitutes a powerful tool to design separation microchips. Since the approach relies on passive mechanisms and is designed to be filter-less, it can be included in a complex architecture and should be particularly suited for in-situ analysis. It is noteworthy that the actual separation size depends on the channels placed after the bifurcation, so that the full system needs to be considered if the downstream part has non-negligible resistance. Given the high resistance of the lateral branch for small sorting size, this is however not too restrictive for sample handling on the lateral, sorted exit.

Our analysis on the influence of Brownian motion shows that the approach is suited down to around $100 \mathrm{~nm}$, for lower sizes approaches exploiting diffusion instead of fighting it, such as Brownian ratchets $^{38}$ or $\mathrm{H}$-filters ${ }^{22}$, should be preferred.

Future work will aim at getting higher efficiency in terms of proportion of filtered fluid extracted, the low fraction of fluid pumped in the filtered, lateral channel being the main weakness of simple hydrodynamic filtration, especially for small sizes. To that purpose, a particularly elegant approach is to focus and concentrate particles close to the sidewall thanks to a series of lateral channels positioned upstream to the sorting one ${ }^{29}$. It is however not suited for the submicron particles aimed in the present work, since the upstream lateral channels must have a critical diameter inferior to the sorted particles, in order to focus, but not extract them. Designing chips with a large number of lateral channels is thus a more direct option. In order to estimate the performance of this approach, let us consider a device with $\mathrm{N}$ lateral outputs, each one designed to correspond to the same sorting size. For simplicity, we consider intersections with equal main channel aspect ratio $\mathrm{r} / \mathrm{w}$, so that equal sorting sizes means equal individual pumping fraction $Q^{*}$. The overall efficiency $Q_{t o t}^{*}$ is defined as the ratio of flow extracted by all the lateral channels to the injected flow. It can be estimated by reasoning on the output (non sorted) fluid: the total flow, $1-Q_{\text {tot }}^{*}$, corresponds to the combination of $\mathrm{N}$ sorting at an efficiency each time $1-Q^{*}$, so that $1-Q_{\text {tot }}^{*}=\left(1-Q^{*}\right)^{N}$. We thus obtain the following relationship between the overall efficiency, the number of branches, and the pumping fractions:

$$
Q_{\text {tot }}^{*}=1-\left(1-Q^{*}\right)^{N} .
$$

For the dimensions considered here, with the general goal to sort submicron particles without any filter effect, $Q^{*} \ll 1$ and $Q_{t o t}^{*} \sim N Q^{*}\left(1-N Q^{*} / 2\right)$. As first approximation, the total efficiency of a device with several lateral channels is thus simply the individual ratio multiplied by the number of channels: $Q_{\text {tot }}^{*} \sim N Q^{*}$, as far as $N Q^{*}$ remains much lower than unity, which corresponds to moderate ratios. Within that limit, each individual lateral channel can be designed thanks to the model presented in the present article, since the flow extracted by all the other lateral channels can be neglected with respect to the main output flow. Equivalently, the hydroelectric scheme presented in Figure 1(c) can be used for each individual channel, the resistances of the other lateral channels leading to negligible modification of flow repartition. As a concrete example, for sorting diameter of $450 \mathrm{~nm}$ (ultrafiltration limit of many conventional filters), for the two-level geometry, lateral channel length is of order $1 \mathrm{~cm}$, and $Q^{*} \sim 0.034 \%$. On a square-centimetre chip, it could seem reasonable to consider a device with a few hundred lateral channels, cm-long with $10 \mu \mathrm{m}$ period (width $5 \mu \mathrm{m}$, spaced by $5 \mu \mathrm{m}$ ), which could 
lead to total efficiency $Q_{t o t}^{*} \sim N Q^{*}$ of order $10 \%$. However, the part of the main output channel that needs to be thin ( $\mathrm{L}^{\prime}$ out in $\left.\S \mathrm{C} .1\right)$ cannot be kept short enough to ensure a low output resistance, so that further design trick would be required. The 3D configuration is better suited to that purpose: it enables more space for lateral channels (since they can be distributed on different layers), and the global resistance of the output channel can be kept reasonably low thanks to increased width. This will be investigated in further study.

More generally, the complete design of a microsystem able to reach an efficiency approaching unity requires a full hydro-electric analogy, since each intersection is in that case not equivalent to the previous one, and the flow repartition at each branching depends on the whole geometry. It would also be worth extending the approach to non-spherical or soft particles, in order to sort according to properties such as shape ${ }^{39}$, deformability ${ }^{40}$ or adhesion.

The sample preparation methodology explained in the present paper is generic and it should apply to sensors, for many areas such as environmental pollution, water quality measurement, as well as in blood microparticles separation.

\section{Acknowledgements}

We acknowledge the RTRA STAE foundation, project MAISOE, and PIA - ANR Digidiag project. , for funding. This work was partly supported by the French RENATECH network.

\section{Notes and references}

1 J. Autebert, B. Coudert, F.-C. Bidard, J.-Y. Pierga, S. Descroix, L. Malaquin and J.-L. Viovy, Methods, 2012, 57, 297-307.

2 A. A. S. Bhagat, H. Bow, H. W. Hou, S. J. Tan, J. Han and C. T. Lim, Med. Biol. Eng. Comput., 2010, 48, 999-1014.

3 D. R. Gossett, W. M. Weaver, A. J. Mach, S. C. Hur, H. T. K. Tse, W. Lee, H. Amini and D. D. Carlo, Anal. Bioanal. Chem., 2010, 397, 3249-3267.

4 D. Di Carlo, D. Irimia, R. G. Tompkins and M. Toner, Proc. Natl. Acad. Sci., 2007, 104, 18892 18897.

5 S.-Y. Yang, S.-K. Hsiung, Y.-C. Hung, C.-M. Chang, T.-L. Liao and G.-B. Lee, Meas. Sci. Technol., 2006, 17, 2001.

6 K. Cheung, S. Gawad and P. Renaud, Cytom. Part J. Int. Soc. Anal. Cytol., 2005, 65, 124-132.

7 A.-E. Saliba, L. Saias, E. Psychari, N. Minc, D. Simon, F.-C. Bidard, C. Mathiot, J.-Y. Pierga, V. Fraisier, J. Salamero, V. Saada, F. Farace, P. Vielh, L. Malaquin and J.-L. Viovy, Proc. Natl. Acad. Sci., 2010, 107, 14524-14529.

8 R. Kwak, S. J. Kim and J. Han, Anal. Chem., 2011, 83, 7348-7355.

9 A. Sarkar and J. Han, Lab. Chip, 2011, 11, 2569-2576.

10 J. L. Probst, A. Messaïtfa, G. Krempp and P. Behra, in Mercury Contaminated Sites, eds. D. R. Ebinghaus, D. R. R. Turner, P. D. L. D. de Lacerda, P. D. O. Vasiliev and P. D. W. Salomons, Springer Berlin Heidelberg, 1999, pp. 501-520.

11 N. S. Barteneva, E. Fasler-Kan, M. Bernimoulin, J. N. Stern, E. D. Ponomarev, L. Duckett and I. A. Vorobjev, BMC Cell Biol., 2013, 14, 23.

12 P. Sajeesh and A. K. Sen, Microfluid. Nanofluidics, 2014, 17, 1-52.

13 A. Lenshof and T. Laurell, Chem. Soc. Rev., 2010, 39, 1203-1217.

14 A. Lenshof, A. Ahmad-Tajudin, K. Järås, A.-M. Swärd-Nilsson, L. Åberg, G. Marko-Varga, J. Malm, H. Lilja and T. Laurell, Anal. Chem., 2009, 81, 6030-6037.

15 M. S. Cheri, H. Latifi, H. Khashei and M. J. Seresht, ELECTROPHORESIS, 2014, 35, 3523-3532.

16 U. Kim, J. Qian, S. A. Kenrick, P. S. Daugherty and H. T. Soh, Anal. Chem., 2008, 80, 8656-8661.

17 T. Zhu, R. Cheng, Y. Liu, J. He and L. Mao, Microfluid. Nanofluidics, 2014, 17, 973-982.

18 M. P. MacDonald, G. C. Spalding and K. Dholakia, Nature, 2003, 426, 421-424. 
19 B. S. Lee, J.-N. Lee, J.-M. Park, J.-G. Lee, S. Kim, Y.-K. Cho and C. Ko, Lab. Chip, 2009, 9, $1548-1555$.

20 J. Steigert, M. Grumann, T. Brenner, L. Riegger, J. Harter, R. Zengerle and J. Ducrée, Lab. Chip, 2006, 6, 1040-1044.

21 B. S. Lee, Y. U. Lee, H.-S. Kim, T.-H. Kim, J. Park, J.-G. Lee, J. Kim, H. Kim, W. G. Lee and Y.K. Cho, Lab. Chip, 2010, 11, 70-78.

22 J. P. Brody, P. Yager, R. E. Goldstein and R. H. Austin, Biophys. J., 1996, 71, 3430-3441.

23 J. P. Brody, T. D. Osborn, F. K. Forster and P. Yager, Sens. Actuators Phys., 1996, 54, 704-708.

24 M. Kim and T. Kim, Analyst, 2013, 138, 6007-6015.

25 J. de Jong, R. G. H. Lammertink and M. Wessling, Lab. Chip, 2006, 6, 1125-1139.

26 J. M. K. Ng, I. Gitlin, A. D. Stroock and G. M. Whitesides, Electrophoresis, 2002, 23, 3461-3473.

27 J. A. Davis, D. W. Inglis, K. J. Morton, D. A. Lawrence, L. R. Huang, S. Y. Chou, J. C. Sturm and R. H. Austin, Proc. Natl. Acad. Sci., 2006, 103, 14779-14784.

28 J. S. McGrath, M. Jimenez and H. L. Bridle, Lab. Chip, 2014, 14, 4139-4158.

29 M. Yamada and M. Seki, Lab. Chip, 2005, 5, 1233-1239.

30 M. Yamada and M. Seki, Anal. Chem., 2006, 78, 1357-1362.

31 H. Bruus, Theoretical Microfluidics, Oxford Master Series in Physics 18, 2007.

32 H. M. Wyss, D. L. Blair, J. F. Morris, H. A. Stone and D. A. Weitz, Phys. Rev. E, 2006, 74, 061402.

33 G. C. Agbangla, P. Bacchin and E. Climent, Soft Matter, 2014, 10, 6303-6315.

34 A. Sauret, E. C. Barney, A. Perro, E. Villermaux, H. A. Stone and E. Dressaire, Appl. Phys. Lett., 2014, 105, 074101.

35 P. Abgrall, C. Lattes, V. Conédéra, X. Dollat, S. Colin and A. M. Gué, J. Micromechanics Microengineering, 2006, 16, 113.

36 R. Courson, S. Cargou, V. Conedera, M. Fouet, M. C. Blatche, C. L. Serpentini and A. M. Gue, RSC Adv., 2014, 4, 54847-54853.

37 D. C. Duffy, J. C. McDonald, O. J. A. Schueller and G. M. Whitesides, Anal. Chem., 1998, 70, 4974-4984.

38 L. R. Huang, E. C. Cox, R. H. Austin and J. C. Sturm, Anal. Chem., 2003, 75, 6963-6967.

39 S. Sugaya, M. Yamada and M. Seki, Biomicrofluidics, 2011, 5, 024103.

40 D. Holmes, G. Whyte, J. Bailey, N. Vergara-Irigaray, A. Ekpenyong, J. Guck and T. Duke, Interface Focus, 2014, 4, 20140011. 